\title{
Repository corticotropin injection in a patient with idiopathic pulmonary fibrosis
}

\begin{abstract}
Immunosuppressants alone or in combination therapy with steroids have been used to treat idiopathic pulmonary fibrosis (IPF) for many years although the supportive clinical data are limited. Furthermore, the adverse effects of steroids and other immunosuppressant are hindering the use of these agents. In the meanwhile, there is much more interests in developing less toxic and more effective pharmacotherapy. Here we report a patient with underlying history of Hypertension, type 2 Diabetes Mellitus, Coronary Artery Disease with angioplasty and stent, Nephrotic Syndrome and Renal Artery Stenosis with stent, Hypercholesterolemia, Hypertriglyceridemia, Hypothyroidism, who developed increasing shortness of breath and deteriorating dyspnea on exertion associated with persistent cough. He was later found to have Idiopathic Pulmonary Fibrosis based on clinical manifestations, roentography and pulmonary parameters. He was initially treated with prednisone for Nephrotic syndrome with progressing improvement in signs and symptoms of co-existing IPF. However, he developed Cushing syndrome due to long-term use of steroids. At that time, he was switched to the treatment of proteinuria with the repository corticotropin injection Acthar Gel (subcutaneous injection $80 \mathrm{mg}$ twice weekly). Incidentally, we found out that there was a significant improvement in follow-up parameters of pulmonary functions and roentography findings, in addition to improvement in proteinuria and renal functions. Therefore, we believe that this medication helps not only to spare the steroid side effects but also works on the direct corticotrophin and some melanocortin receptors in renal and pulmonary systems thus may contribute to the significant improvement in reducing the signs and symptoms of IPF as well as proteinuria due to Nephrotic syndrome.
\end{abstract}

Keywords: acthar, idiopathic pulmonary fibrosis

\section{Introduction}

Idiopathic pulmonary fibrosis (IPF) is a type of chronic interstitial lung disease characterized by a progressive and irreversible decline in lung function. Patients typically present with gradual onset of shortness of breath and dry cough developing over several months. Even though the cause is left unknown, there are many well recognized risk factors like cigarette smoking, certain viral infections and some potential risk factors including gastroesophageal reflux disease (GERD), or genetic predisposition (familial IPF). ${ }^{1}$ However, not all people with IPF have these risk factors and therefore do not provide a completely satisfactory explanation for the disease. It is possible that multiple micro injuries to alveolar epithelial cells induce a fibrotic environment, and that growth factors secreted by the injured epithelial cells recruit fibroblasts. These fibroblasts transform into myofibroblasts which express features of both fibroblasts and smooth muscle cells. These myofibroblasts secrete collagen which accumulates due to imbalance between interstitial collagenases and their tissue inhibitors. The end result is a complex reticulum of fibrous tissues that is highly interconnected and extends from pleura into the underlying parenchyma. ${ }^{2}$

Diagnosis of IPF needs to be supported by a high-resolution CT scan or lung biopsy or both and also requires ruling out other potential causes of interstitial lung disease. Once the diagnosis is established with high degree of confidence, therapy is initiated according to severity of the disease. Unfortunately, no medicine has been found to cure IPF. ${ }^{3}$ Management of IPF includes a wide range of Medications (Newly available monotherapy of Pirfenidone / Nintedanib and Older regime of combined Prednisone, azathioprine, and $\mathrm{N}$ - acetylcysteine or monotherapy with $\mathrm{N}$-acetylcysteine) and supportive care (supplemental oxygen, pulmonary rehabilitation, seasonal influenza and pneumococcal vaccination) which have been
Volume 6 Issue 5 - 2018

\author{
Alexander M Swan,' Cho Nwe Aye, ${ }^{2}$ Chan \\ Nyein Thida, ${ }^{2}$ Pyae Sone Htwe, ${ }^{2}$ Thar Sann \\ Oo, ${ }^{2}$ Moe Thuza, ${ }^{2}$ Nway Nway, ${ }^{2}$ San Oo \\ Lwin, ${ }^{2}$ Htet Htet Kyaw, ${ }^{2}$ Zaw Nyein Aye, ${ }^{2}$ \\ San Myat Htun, ${ }^{2}$ May Thu Zin ${ }^{2}$ \\ 'AOA Professor of Medicine \& Clinical Faculty, Rutgers New \\ Jersey Medical School, USA \\ ${ }^{2}$ Nephrology Hypertension Renal transplant and Renal Therapy, \\ USA
}

Correspondence: AOA Professor of Medicine \& Clinical Faculty, Rutgers New Jersey Medical School, 185 S Orange Ave, Newark, NJ, 07 I03I; Tel 732-750-5555, Fax 732-750-5550, Email alexmswan@gmail.com

Received: November 29, 2018 | Published: December 27,
2018 2018 used either alone or in combination. The last solution after above are failed to respond may be lung transplantation which is an option for a patient with progressive decline in lung function and minimal comorbidities. ${ }^{2}$ Prednisone showed a promising improvement in this patient's symptoms but there were potential adverse effects of steroid like Cushing syndrome and proteinuria secondary to steroid induced diabetes nephropathy.

Repository corticortropin injection has been recently used to treat proteinuria effectively. ACTH hormone is cleaved from its pro-opiomelanocortin (POMC) prohormone as are $\alpha$-melanocytestimulating hormone $(\alpha-\mathrm{MSH}), \beta-\mathrm{MSH}$ and $\gamma-\mathrm{MSH}$. ACTH and $\alpha-, \beta$ - and $\gamma$-MSH comprise the melanocortins. This drug is a slow-release formulation of full-sequence ACTH (1-39) (80units/ $\mathrm{ml}$ ) and contains additional biologically active POMC peptides which bind to and are believed to activate melanocortin receptors (MCRs). There are five melanocortin receptors (MCRs) in the body. ACTH binds to all of these MCRs with only MC2R being implicated in adrenal steroidogenesis. ${ }^{4}$ MCRs are molecules produced in response to inflammation and can contribute to controlling and balancing the inflammatory process by exerting anti-inflammatory actions. We found out those anti-inflammatory actions can also be used in respiratory disease including pulmonary fibrosis, just like in this case, without suffering from common adverse reactions such as fluid retention, alteration in glucose tolerance, elevation in blood pressure, behavior and mood changes, increased appetite and weight gain.

\section{Case description}

A 73-year-old Asian male with uncontrolled resistant labile hypertension was referred to the clinic from PCP in 2011. The patient 
was given an intensive care and a stepwise approach with multiple antihypertensive drugs to control his hypertension. After that, the blood pressure was stable and it was successfully controlled within certain range with medications. Later afterwards, he complained of color changes in isolated three fingers in right hand, and then the complete workup was done. According to the lab results and Imaging, he was diagnosed as Essential Hypertension which is salt sensitive and Unilateral Renal Artery stenosis. Even after surgical correction of the stenosis with stent, the patient still needed to take multiple medications for his BP control. However, his Creatinine was reduced and his renal function was improved by $15 \%$ after the surgical procedure. On later months, his sugar level was high and there was proteinuria in urine analysis as well as hypercholesterolemia. Even though he was treated with proper medications and controlled diet, his proteinuria was not improved at that time. Therefore, Complete Proteinuria workups such as Complement active C3, C4 and Hep A, B, C, serum protease $3 \operatorname{IgG}$, Cryoglobulin, Lupus Anticoagulant, ANA, Anti dsDNA, RF, P-ANCA and Fibronectin IgA were done. The tests were all negative and we assume that this is FSGS and sent him for renal biopsy. The biopsy result showed that it is FSGS and then, he was treated with prednisolone. In the meanwhile, he developed SOB and non-productive cough for a couple of months in 2017. Later, he was diagnosed with pneumonia and treated with antibiotics. After complete course of antibiotics, he still had SOB and dry cough which progressively worsened. In those months, his fever was off and on and cough became productive. Therefore, chest $\mathrm{CT}$ was ordered and we found out that CT result described pulmonary fibrosis. At that time, he was given high dose of prednisolone for proteinuria and lung symptoms. After a few weeks, he got better with prednisolone. As the long-term steroid use worsened his sugar control, proteinuria, truncal obesity and later he developed Cushion features. Eventually, the use of steroid was tapered off and, luckily, this repository corticotrophin injection was released around that time. The patient agreed to get treated with this medication and his proteinuria, dry cough and SOB were gradually improving while his sugar level, cholesterol level and $\mathrm{BP}$ were able to manage within controlled range. After one- month treatment, his Cushing syndrome and proteinuria started to improve. He did not complain of any respiratory symptoms and his PFTs done three months later turned out to be within normal limit. At that time his lab reports showed protein/creatinine ratio $175 \mathrm{mg} / \mathrm{g}$ creatine and $\mathrm{HbA1C} 9.1 \%$ and the other lab results were within normal range. While he was on this drug, his DM was aggressively treated with combination of Trulicity (GLP-1 inhibitor) injection twice weekly, Trajenta (DDPV inhibitor) 5mg daily and short acting Insulin as needed as. Furthermore, he was given statins for his lipid control.

The following figures compare the patients pulmonary function tests before and after the Acthar injection (Figure 1-3).

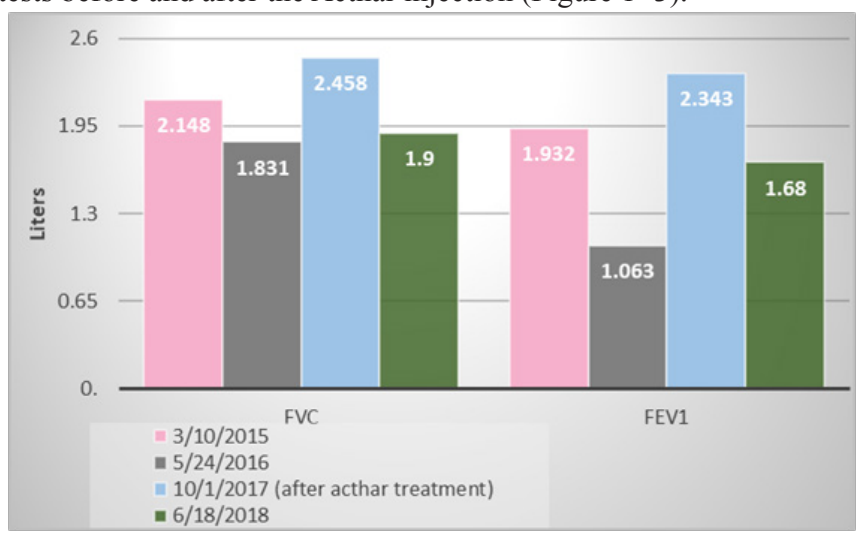

Figure I Comparison of FVC and FEVI Values before and after Starting Acthar Injection.

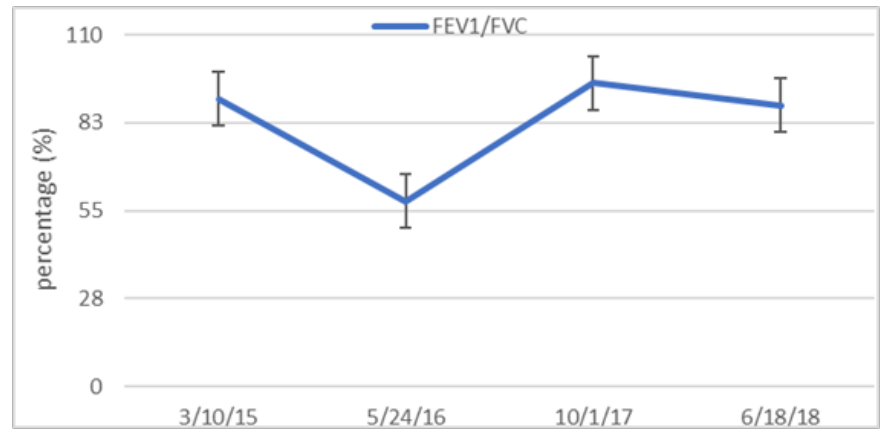

Figure 2 Changes in FEVI/FVC after Starting Acthar Injection.

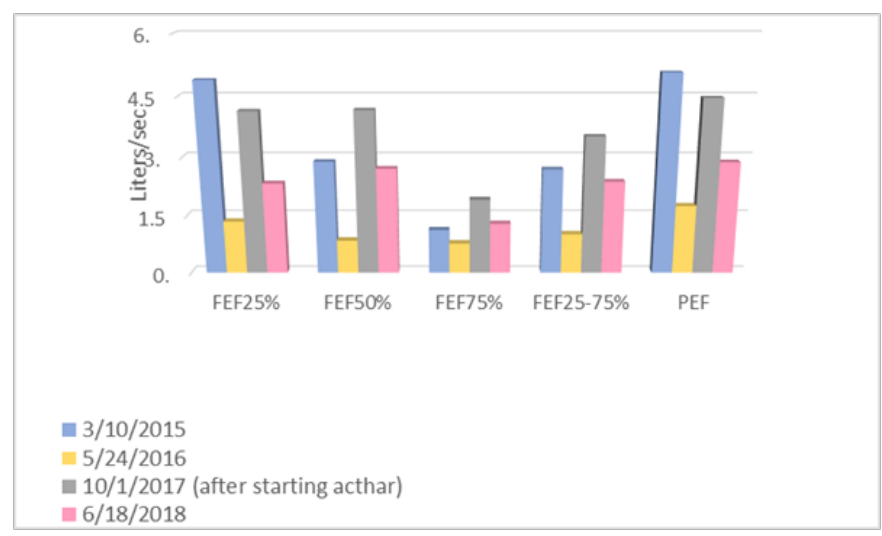

Figure 3 Comparisons of Pulmonary Function Test Results Before and After Weekly Acthar Injections.

\section{Discussion}

In this case presentation, there is a variety of treatment options for IPF and steroid may initially be beneficial for this patient although it has long-term multiple side effects. All available managements with medications are found to be unsatisfactory result for better curative power in treatment of IPF. According to our case, this repository corticortropin injection could be useful in managing patient with IPF and it may be a potentially successful therapeutic drug of choice in the coming days. Therefore, further studies and research should be done to explore the efficiency and efficacy of the potential therapeutic effects of Repository Corticotropin in the future.

\section{Conclusion}

This case report highlights that the therapeutic effects of Repository Corticotropin on melanocortin receptors in addition to its glucocorticoid effects in renal and pulmonary systems. These should be observed in treatment of Nephrotic syndrome and IPF.

\section{Acknowledgments}

Contributors: Dr Alexandar M Swan acts as the guarantor and supervisor. Cho Nwe Aye contributed in writing abstract and introduction, Chan Nyein Thida in gathering information and discussion, Pyae Sone Htwe in case description and providing figures, Thar Sann Oo in conclusion, manuscript editing. All authors approved the final version of this manuscript.

\section{Conflicts of interest}

Authors declare there is no conflicts of interest. 


\section{References}

1. Raghu G, Collard HR, Egan JJ, et al. An Official ATS/ERS/JRS ALAT Statement: Idiopathic Pulmonary Fibrosis: Evidence-based Guidelines for Diagnosis and Management. Am J Respir Crit Care Med. 2011;183(6):788-824.

2. Selman M, King TE, Pardo A, et al. Idiopathic pulmonary fibrosis: prevailing and evolving hypotheses about its pathogenesis and implications for therapy. Ann Intern Med. 2001;134(2):136.

3. Idiopathic Pulmonary Fibrosis. Cleveland clinic diagnosis, tests, management and treatment.

4. Catania A, Gatti S, Colombo G, et al.Targeting melanocortin receptors as a novel strategy to control inflammation. Pharmacol Rev. 2004;56(1):1-29. 